\title{
LIMITS IN TERMS OF ORDER, WITH EXAMPLE OF LIMITING ELEMENT NOT APPROACHABLE BY A SEQUENCE*
}

BY

\author{
RALPH E. ROOT
}

\section{INTRODUCTION.}

The importance of the rôle of the order relation in the theory of the linear continuum has been recognized since the appearance, in 1872, of Dedekind's Stetigkeit und irrationale Zahlen, $\dagger$ and it received added importance at the hands of Cantor $\ddagger$ in 1895. A later contribution to the theory was made by 0 . Veblen $\S$ in 1905, and a systematic account of the theory of the continuum as a type of order was given by $\mathrm{E}$. V. Huntington $\|$ the same year. The work of these writers is directed toward a complete characterization of the linear continuum in terms of order alone. A set or class of elements, otherwise undefined, is assumed to fulfil conditions, stated in terms of order, sufficiently restrictive to admit of effective use of the class in the rôle of range of the continuous independent variable.

Meanwhile certain classes have been recognized as being, in effect, the range of an independent variable, while not fulfilling all the conditions of the linear continuum. The desirability of utilizing analogies that exist between theories that pertain to these classes and the theory of the continuum is obvious. To this end it is important to generalize, as far as may be, the notions of point sets so as to be applicable to such classes.

The thesis of $M$. Fréchet $\uparrow$ was a notable effort toward this form of generalization. He presupposes a definition of limit of a sequence of elements, the definition fulfilling certain mild conditions, and defines in terms of this the notion of limiting element of a subclass, and develops a theory applicable to any class for which a limit relation can be defined fulfilling the prescribed

\footnotetext{
* Presented to the Society (Chicago), March 21, 1913.

† English translation by W. W. Beman, in a volume titled Dedekind's Essays on the Theory of Numbers, 1901.

$\ddagger$ Mathematische Annalen, vol. 46 (1895), p. 481.

\$ These Transactions, vol. 6 (1905), p. 165.

\|A n a l s of Mathematic s, ser. 2, vol. 6 (1905), p. 151, and vol. 7 (1905), p. 15.

TRendicontidel Circolo Matematico di Palermo, vol. 22 (1906), p. 6.
} 
conditions. He later adopts an analogue of the distance function (voisinage), the restrictions of which give a much more extensive theory.

E. H. Moore, in his paper at the Rome Congress in $1908,{ }^{*}$ and in his later published work on general analysis, $\uparrow$ introduces a form of generalization of different character, considering properties of classes whose elements are real valued functions of a variable absolutely unconditioned. This general variable admits of a wide range of special applications.

E. R. Hedrick $\ddagger$ contributes to the theory of such general classes by defining, in terms of the limit relation used by Fréchet, an " enclosable property" which replaces the voisinage or distance function used by the latter to define a special type of limit. In a paper by $T$. H. Hildebrandt $\S$ in which the voisinage of Fréchet is replaced by a $K$ relation of a type used by $E$. H. Moore, it is shown that most of the theorems proved by Fréchet may be based on milder assumptions than he used. The papers by Fréchet, Hedrick and Hildebrandt each consists of two stages, the first stage pertaining to a range possessing a mildly conditioned definition of limit of a sequence, the second stage pertaining to a range conditioned in such manner as to involve a notion closely analogous to that of " neighborhood," and introducing in some form the notion of " uniformity on the range."

In a former paper $\|$ by the present writer postulates are stated directly in terms of "neighborhnod," but are free from any requirement of uniformity on the range. This absence of uniformity facilitates the treatment of ideal limiting elements, while invariance of postulates under the process of composition of classes is utilized to simplify the treatment of multiple and iterated limits. The paper may, in some sense, be regarded as an intermediate stage between the two stages of each of the three papers just mentioned.

The object of the present paper is to use in this field of generalization some of the simple properties of ordered classes. A triadic relation of the type of " betweenness" serves in the definition of segment, which, with certain mild postulates that are fulfilled by any simply ordered class, is effective in the development of a theory of limits and continuity. The postulates possess the invariance under composition and the freedom from uniformity that are

* On a Form of General Analysis, with Application to Linear Differential and Integral Equations, Atti del $I^{\circ}$ Congresso Internazionale dei Matematici, Roma, 1808, vol. 2, p. 98.

† Introduction to a Form of General Analysis, published in a volume titled The New Haven Mathematical Colloquium, 1910.

$\ddagger$ On Properties of a Domain for which any Derived Set is Closed, these T r a n a c t i o $\mathrm{n}$, vol. 12 (1911), pp. 285-294.

8 A Contribution to the Foundations of Frechet's Calcul Fonctionnel, A m e r i c a n J o u r na I of Mathematics, vol. 34 (1912), p. 237.

II Iterated Limits in General Analysis, American J o r nal of Mathematica, Jan. and Apr., 1914. 
emphasized in the previous paper, and avoid some of the restrictions of that paper.

The theory developed in sections 2-7 may be characterized as a " neighborhood theory," being based on a definition of limiting element in terms of segment, a type of neighborhood. In section 8 we indicate the character of a " sequential theory"* based on the same postulates, which emerges by the adoption of a definition of limiting element in terms of "limit of a sequence," as is done in the first stage of the paper by Fréchet. This form of definition is used also by Hedrick, and in the first part of the paper by Hildebrandt. Finally, in section 9, we define a " sequential property " for classes of elements, the presence of which, in addition to Postulates I-III, is a necessary and sufficient condition for the equivalence of the two theories considered. It is shown that simply ordered classes exist which do not possess this property. $\dagger$ A fourth postulate, sufficient to secure the sequential property, is discussed in relation to certain fundamental assumptions that have occurred in discussions of the linear continuum and of general analysis.

\section{Definitions in a $\operatorname{system}(\mathscr{Q} ; B)$.}

The system $(\mathfrak{Q} ; B)$ with which we are concerned consists of a class $\mathfrak{Q}$ of elements $q$ of any kind whatever, and a relation $B$ defined for triads of elements of $\mathfrak{Q}$. The relation $B$ is said to be defined for a class $\mathfrak{Q}$ if for every three elements $q_{1}, q_{2}$, and $q_{3}$ (distinct or not) the question "does the relation $B_{q_{1} q_{3} q_{2}}$ hold " has a definite answer, yes or no. A simple instance of such a system is obtained if we take for $\mathfrak{Q}$ the class of all points on a line, and let $B$ denote the ordinary relation of "betweenness," so that $B_{q_{1} q_{2} q_{3}}$ may be read " $q_{2}$ is between $q_{1}$ and $q_{3}$. "

The following definitions are found convenient in developing a theory pertaining to a system $(\mathbb{Q} ; B)$.

Definition 1. If $q_{1}$ and $q_{2}$ are such that $B_{q_{1} q_{3}}$ holds for some $q$, the class of all elements $q$ such that $B_{q_{1} q_{2}}$ holds is called a segment.

Such a segment is denoted by $\widetilde{S}_{q_{1} q_{2}}$ or, in case clearness permits, simply by $\mathfrak{S}$.

* T. H. Hildebrandt (loc. cit., p. 239) uses the term "sequential continuity" with respect to functions on a range where limiting element has a definition of this form.

†In the Bulletin of the American Mathematical Society, vol. 17, (1911), p. 538, we gave a brief forecast of the principal features of the paper cited above on iterated limits. The closing sentence of that forecast states that the treatment is available for any class having linear order. The statement is exact, but the application then in view would give a theory for linear sets substantially equivalent to the "sequential" theory for such sets indicated in the present paper. This result is attained by assigning to each element not approachable by a sequence a neighborhood consisting of the element itself, thus making it an isolated element also in the neighborhood theory. In the last section of the present paper we indicate a restriction on linear sets sufficient to render the neighborhood theory of this paper a special case under the general theory of the paper cited above. 
Defrnirion 2. An element $q$ is a limiting element of a subclass $\mathfrak{B}$ of $\mathfrak{Q}$ if every segment containing $q$ contains an element $p$ of $\mathfrak{B}$ distinct from $q$.

Defrnimion 3. A subclass $\mathfrak{B}$ is closed if every limiting element of $\mathfrak{B}$ is in $\mathfrak{P}$.

Defnnimion 4. A subclass $\$$ is dense in itself if every element of $\$$ is a limiting element of $\mathfrak{P}$.

Derintrion 5. A subclass $\mathfrak{B}$ is compact if every infinite subclass of $\mathfrak{P}$ has a limiting element.*

Defrnition 6. An element $q$ is interior to a subclass $\mathfrak{B}$ if there is a segment containing $q$ and contained in $\mathfrak{P}$.

Derminimon 7. An element $q$ is a limit of a sequence $\left\{q_{n}\right\}$ if for every segment $\mathfrak{S}$ containing $q$ there is an $n_{6}$ such that, for $n>n_{\odot}, q_{n}$ is in $\mathfrak{S}$.

Defintrion 8. The derived class of a class $\mathfrak{B}$ is the class of all limiting elements of $\mathfrak{B}$.

Definition 9. The frontier class of a class $\mathfrak{B}$ is the class of all elements in $\mathfrak{B}$ or the derived class of $\mathfrak{B}$ that are not interior to $\mathfrak{P}$.

\section{THEOREMS REQUIRING NO RESTRICTIONS ON THE SYSTEM.}

The theorems of the present section hold for any system of the type $(\mathfrak{Q} ; B)$ in the absence of any restrictions whatever. $t$ The proofs, being entirely obvious, are omitted.

ThEoREM I. If $\mathfrak{R}$ is the derived class of $\mathfrak{B}$, then the class $\mathfrak{B}+\mathfrak{R}$ is closed.

THEOREM II. The frontier class of any class is closed.

TheOREM III. If $q$ is a limiting element of $a$ subclass $\mathfrak{B}$ it is a limiting element of every class containing $\mathfrak{B}$. $\ddagger$

Theorem IV. $A$ class $\mathfrak{B}$ is compact if and only if every subclass of $\mathfrak{B}$ is compact. $\$$

Theorem V. A class formed of a finite number of compact classes is compact.

Theorem VI. A sequence formed by repeating a single element has that element as limit.

Theorem VII. If a sequence has a limit every infinite subsequence has the same limit.

- Fréchet, loc. cit., p. 6.

t It is common, under similar circumstances, to state theorems as corollaries of definitions. We desire to emphasize the fact that the office of a definition is to conserve economy in the use of language, and that it is, in reality, a part of the statement of every proposition which involves the term it defines. The validity of a theorem depends on the definitions of its terms only in the same sense that it depends on its own hypothesis.

I A proposition of this form is used by F. Riess as a postulate in his paper on Stetiokeitsbegriff und abstracte Mengenlehre, At i del IV Congresso Internazionale dei Matematici, Roma, 1808, vol. 2, pp. 18-24. Propositions of the form of his other postulates occur as theorems in section 7 .

8 This theorem and the next are given by Fr6chet, loc. cit., p. 7. Theorems VI and VII are aimilar in form to postulates used by Frochet. Further reference to his work is made in section 7. 
It should be understood that the limit of a sequence need not be unique. We are able to prove this property only after the adoption of Postulate III.

THEOREM VIII. If $q$ is interior to $\$$, all but a finits number of terms of any sequence whose limit is $q$ are interior to $\mathfrak{B} .^{*}$

Theorem IX. If there exists a sequence of distinct elements of $\mathfrak{B}$ having $q$ as limit, $q$ is a limiting element of $\mathfrak{B}$.

We note that the converse of this proposition holds only in a very restricted system. It is because of this that we find a divergence of the " neighborhood" and the "sequential " theories. We discuss this question more fully in the last two sections.

\section{Theorems in a composite system.}

From two systems $\left(Q^{\prime} ; B^{\prime}\right)$ and $\left(Q^{\prime \prime} ; B^{\prime \prime}\right)$ of the type under consideration we derive a system $(\mathfrak{Q} ; B)$ of the same type. The class $\mathfrak{Q}$ is the product class $\mathfrak{Q}^{\prime} \mathfrak{Q}^{\prime \prime}$, consisting of all elements $q$ of the form $q=q^{\prime} q^{\prime \prime}$, where $q^{\prime}$ is in $\mathfrak{Q}^{\prime}$ and $q^{\prime \prime}$ is in $\mathfrak{Q}^{\prime \prime}$. The element $q$ is not to be regarded as a product, but simply as the pair $q^{\prime} q^{\prime \prime}$. For three elements $q_{1}, q_{2}, q_{3}$ the relation $B_{q_{1} q_{3} q_{3}}$ holds if and only if $q_{1}=q_{1}^{\prime} q_{1}^{\prime \prime}, q_{2}=q_{2}^{\prime} q_{2}^{\prime \prime}$ and $q_{3}=q_{3}^{\prime} q_{3}^{\prime \prime}$, and the relations $B_{q_{1}^{\prime} \text { 'rqqe }}^{\prime}$

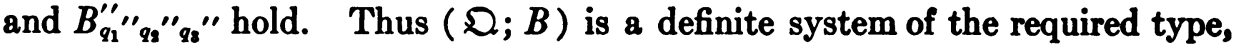
and is called the composite system of the systems $\left(\mathfrak{Q}^{\prime} ; B^{\prime}\right)$ and $\left(\mathfrak{Q}^{\prime \prime} ; B^{\prime \prime}\right)$. Similarly any number of systems of this type give rise to a definite composite system. $\dagger$

A system $(\mathfrak{Q} ; B)$ may fulfil the following postulate:

Postulate I. If $q$ is an element of $\mathfrak{Q}$ there exists a segment containing $q$. If the relation $B$ is interpreted as ordinary betweenness, and fulfils conditions sufficient to render $\mathfrak{Q}$ a simply ordered class, then the addition of this postulate is equivalent to the assumption that there is no first or last element. This is not, in effect, any restriction; for any simply ordered class may be regarded as a subclass of a class having no extreme elements. Moreover, if we so modify the relation $B$ that $B_{q q q_{1}}$ and $B_{q_{1} q q}$ shall hold in case $q$ is an extreme element, while it remains otherwise as ordinary betweenness, then we find that Postulate $I$ is no restriction on a simply ordered class.

If each of two or more systems fulfil Postulate I their composite system does also. And if a composite system fulfils this postulate so must every component system, providing that $\mathfrak{Q}$ in the composite system contains at least one element. Excluding the trivial case just provided against, we may say that Postulate $I$ is invariant under composition of systems.

The theorems of this section are, for the sake of clearness and brevity, stated for a system $(\mathfrak{Q} ; B)$, composite of two systems, $\left(\mathfrak{Q}^{\prime} ; B^{\prime}\right)$ and $\left(Q^{\prime \prime} ; B^{\prime \prime}\right)$.

* E. R. Hedrick, loc. cit., p. 286.

† Compare T. H. Hildebrandt, loc. cit., p. 250. 
The notations $\mathfrak{B}, \Re$, etc., indicate subclasses of $\mathfrak{Q}$, and with suitable accents they denote subclasses of $\mathfrak{Q}^{\prime}$ and $\mathfrak{Q}^{\prime \prime}$. The manner of extending to the case of a composite system of several systems is entirely obvious.

Theorem X. A class $\mathfrak{P}$ is a segment if and only if there exist segments $\mathfrak{S}^{\prime}$ and $\mathfrak{S}^{\prime \prime}$ such that $\mathfrak{B}$ is the product class $\mathfrak{S}^{\prime} \mathfrak{S}^{\prime \prime}$.

This proposition is obviously true, even for systems in which Postulate I is not assumed to hold.

THeOREM XI. If $\mathfrak{B}$ is the product class $\mathfrak{B}^{\prime} \mathfrak{B}^{\prime \prime}$ and if $q=q^{\prime} q^{\prime \prime}$, then a necessary and sufficient condition that $q$ shall be a limiting element of $\mathfrak{B}$ is that $q^{\prime}$ is a limiting element of $\mathfrak{B}^{\prime}$ and $q^{\prime \prime}$ is in $\mathfrak{B}^{\prime \prime}$, or $q^{\prime \prime}$ is a limiting element of $\mathfrak{P}^{\prime \prime}$ and $q^{\prime}$ is in $\mathfrak{P}^{\prime}$, or $q^{\prime}$ and $q^{\prime \prime}$ are limiting elements respectively of $\mathfrak{B}^{\prime}$ and $\mathfrak{P}^{\prime \prime}$.

That the condition is sufficient is easily seen, without the use of the postulate. The necessity of the condition may be shown to involve the postulate as follows: Let $q^{\prime}$ be in a segment containing no other element, and suppose $q^{\prime \prime}$ is not in any segment; then $q$ is not in any segment and is therefore a limiting element of every $\mathfrak{B}$, but $q^{\prime}$ is not a limiting element of any $\mathfrak{B}^{\prime}$. By considering $\mathfrak{B}=\mathfrak{B}^{\prime} \mathfrak{P}^{\prime \prime}$, where $\mathfrak{P}^{\prime}$ does not contain $q^{\prime}$, we see that the condition is not necessary. The proof of the necessity of the condition is very simple if Postulate I is fulfilled.

Theorem XII. If $\mathfrak{P}=\mathfrak{P}^{\prime} \mathfrak{P}^{\prime \prime}$, then $\mathfrak{P}$ is closed if both $\mathfrak{P}^{\prime}$ and $\mathfrak{P}^{\prime \prime}$ are closed, and, in case $\mathfrak{P}$ contains at least one element, both $\mathfrak{P}^{\prime}$ and $\mathfrak{B}^{\prime \prime}$ are closed if $\mathfrak{B}$ is closed.

The first part of this theorem involves the necessity of the condition in Theorem XI, and therefore the use of the postulate, but the second part of the theorem involves only the sufficiency of the condition, and can be proved for an unrestricted system.

ThEOREM XIII. If $\mathfrak{B}=\mathfrak{B}^{\prime} \mathfrak{P}^{\prime \prime}$, then $\mathfrak{B}$ is dense in itself if and only if one of the component classes, $\mathfrak{P}^{\prime}$ or $\mathfrak{B}^{\prime \prime}$, is dense in itself.

The proof may be arranged, without use of the postulate, as follows: Suppose $\mathfrak{B}^{\prime}$ is dense in itself. An element $p$ is of the form $p^{\prime} p^{\prime \prime}$, and a segment $\mathfrak{S}$ containing $p$ is of the form $\mathfrak{S}^{\prime} \mathfrak{S}^{\prime \prime}$, where $\mathfrak{S}^{\prime}$ contains $p^{\prime}$ and $\mathfrak{S}^{\prime \prime}$ contains $p^{\prime \prime}$. $\mathfrak{S}^{\prime}$ contains a $p_{1}^{\prime}$ distinct from $p^{\prime}$, and $p_{1}=p_{1}^{\prime} p^{\prime \prime}$ is in $\subseteq$ and distinct from $p$. Thus every $p$ is seen to be a limiting element of $\mathfrak{B}$, and $\mathfrak{B}$ is dense in itself. Now suppose neither $\mathfrak{P}^{\prime}$ nor $\mathfrak{B}^{\prime \prime}$ is dense in itself, then there exists $p^{\prime}$ in a segment $\widetilde{S}^{\prime}$ containing no other element of $\mathfrak{P}^{\prime}$, and there exists $p^{\prime \prime}$ in a segment $\mathfrak{S}^{\prime \prime}$ containing no other element of $\mathfrak{P}^{\prime \prime}$, thus the element $p=p^{\prime} p^{\prime \prime}$ is in the segment $\mathfrak{S}=\mathfrak{S}^{\prime} \mathfrak{S}^{\prime \prime}$ which contains no other element of $\mathfrak{B}$. This element $p$ is not a limiting element of $\mathfrak{P}$, so that $\mathfrak{P}$ is not dense in itself.

Theorem XIV. (a) If $\mathfrak{B}=\mathfrak{B}^{\prime} \mathfrak{P}^{\prime \prime}$, then if $\mathfrak{B}$ is compact both $\mathfrak{P}^{\prime}$ and $\mathfrak{P}^{\prime \prime}$ are compact, provided that each contains at least one element.

(b) If $\mathfrak{B}=\mathfrak{B}^{\prime} \mathfrak{P}^{\prime \prime}$ and $\mathfrak{B}^{\prime}$ and $\mathfrak{P}^{\prime \prime}$ are both compact, then every infinite product class in $\mathfrak{B}$ has a limiting element. 
The proof of $(a)$ requires the use of Postulate I, but (b) holds for any system. It will be observed that the compactness of the two component classes does not imply the compactness of $\mathfrak{B}$, except in case of a more restricted system.*

TheOReM XV. If $\mathfrak{B}=\mathfrak{B}^{\prime} \mathfrak{B}^{\prime \prime}$ and $q=q^{\prime} q^{\prime \prime}$, then $q$ is interior to $\mathfrak{P}$ if and only if $q^{\prime}$ and $q^{\prime \prime}$ are interior to $\mathfrak{B}^{\prime}$ and $\mathfrak{P}^{\prime \prime}$ respectively.

This theorem clearly does not involve Postulate $\mathrm{I}$.

THEOREM XVI. If $q=q^{\prime} q^{\prime \prime}$, then a necessary and sufficient condition that $q$ shall be the limit of the sequence $\left\{q_{n}\right\}$ is that the sequences $\left\{q_{n}^{\prime}\right\}$ and $\left\{q_{n}^{\prime \prime}\right\}$, where $q_{n}=q_{n}^{\prime} q_{n}^{\prime \prime}$ for every $n$, shall have respectively the limits $q^{\prime}$ and $q^{\prime \prime}$.

As in Theorem XI, the necessity of the condition involves the postulate, while the sufficiency can be proved for an unrestricted system. The proof, being very simple, is omitted.

\section{Limits AND CONTINUITY OF FUNCTIONS.}

Functions defined on subclasses of $\mathfrak{Q}$ are denoted by the letters $\mu, \varphi, \theta$, etc. A function $\mu$ is said to be defined on a subclass $\mathfrak{B}$ if to every element $p$ there corresponds a definite function value $\mu_{p}$, which is a real number, or $+\infty$ or $-\infty$. With respect to a given function $\mu$ on a given class $\mathfrak{B}$ we consider, relative to a definite limiting element $l$ of $\mathfrak{P}$, three symbols,

$$
\lim _{p \rightarrow i} \mu_{p}, \varlimsup_{p \rightarrow i} \mu_{p}, \lim _{p \rightarrow l} \mu_{p},
$$

which denote respectively the limit, the upper limit and the lower limit of $\mu$ as $p$ approaches $l$. In this section 5 , the symbol $p \rightarrow l$ under " lim" is omitted; it is to be understood in every case that we mean the limit as $p$ approaches $l$.

Definition 10. Following are the conditions under which the limit, the upper limit and the lower limit of $\mu$ on $\mathfrak{B}$ exist at a given limiting element $l$.

(a) $\lim \mu_{p}=a$ is equivalent to the condition: For every $e$ there exists a segment $\mathfrak{S}_{e}$ containing $l$ such that for every $p$ in $\mathfrak{S}_{e}$ distinct from $l$ we have $\left|\mu_{p}-a\right| \leqq e . \dagger$

(b) $\varlimsup \lim \mu_{p}=a$ is equivalent to the two conditions: (1) For every $e$ there exists an $\mathfrak{S}_{0}$ containing $l$ such that if $p$ is in $\mathfrak{S}_{e}$ and distinct from $l$ then $\mu_{p} \leqq a+e$. (2) For every $\mathfrak{S}$ containing $l$ and every $e$ there exists a $p$ in $\mathfrak{S}$ distinct from $l$ such that $\mu_{p} \geqq a-e$.

(c) $\varlimsup \lim \mu_{p}=+\infty$ is equivalent to the condition: For every $\subseteq$ containing $l$ and every $a$ there is a $p$ in $\subseteq$ distinct from $l$ such that $\mu_{p}>a$.

* See Theorem VIII (d) of chapter II of the paper on Iterated Limits in General Analysis, loc. cit. In section 9 of the present paper conditions sufficient to validate such a theorem are stated for a system $(D ; B)$.

$\dagger$ The letter $a$ denotes a real number, while $e$ invariably denotes a positive real number. 
(d) $\varlimsup \mu_{p}=-\infty$ and $\lim \mu_{p}=-\infty$ are each equivalent to the condition: For every $a$ there exists an $\mathfrak{S}_{a}$ containing $l$ such that if $p$ is in $\mathfrak{S}_{a}$ and distinct from $l$ then $\mu_{p}<a$.

(e) Conditions for $\lim \mu_{p}=a, \lim \mu_{p}=-\infty$ are analogous to conditions in (b) and (c), while conditions for $\lim \mu_{p}=+\infty$ and $\lim \mu_{p}=+\infty$ are analogous to $(d)$.

Theorem XVII. If a function $\mu$ defined on $\mathfrak{B}$ is such that for a given limiting element $l$ of $\mathfrak{B}$ there exists an a such that for every $p$ distinct from $l$ we have $\left|\mu_{p}\right| \leqq a$, then there exist uniquely $\bar{a}$ and $\underline{a}$ such that

$$
\varlimsup_{p \rightarrow l} \mu_{p}=\bar{a}, \varliminf_{p \rightarrow l} \mu_{p}=\underline{a} .
$$

For each $\subseteq$ containing $l$ let $\bar{a}_{\tilde{E}}$ be the least upper bound of $\mu_{p}$ for all elements $p$ in $\subseteq$ distinct from $l$. It is not difficult to see that the greatest lower bound of $\bar{a}_{\mathfrak{E}}$ for all segments $\mathfrak{S}$ containing $l$ is the $\bar{a}$ required. Similarly the existence of $\underline{a}$ may be shown. The proof depends on Postulate I.

If in the hypothesis of this theorem we remove the restriction that $\mu$ is bounded on the range obtained by omitting the element $l$ from $\mathfrak{P}$, then the least upper bound of $\mu_{p}$ on a given $\widetilde{S}$, excluding $p=l$, may be $+\infty$ or $-\infty$, so that the greatest lower bound of these least upper bounds may be $+\infty$ or $-\infty$. But in any case the latter quantity definitely exists, and is the upper limit of $\mu$ at $l$. Since a similar procedure gives the lower limit of $\mu$ at $l$, we have the following corollary.

CoRollary. For every $\mu$ defined on $\mathfrak{B}$ and for every limiting element $l$ of $\mathfrak{B}$ the upper limit of $\mu$ at $l$ and the lower limit of $\mu$ at $l$ exist.

But making use of Postulate I it is not possible to prove that $\overline{\lim } \mu_{p} \geqq \lim \mu_{p}$, and the existence of a limit for $\mu$ at a limiting element $l$ neither implies nor is implied by the equality of the upper and lower limits of $\mu$ at $l$. Furthermore, the limit of a function at a limiting element, when it exists, is not necessarily unique. These points may be established by the following examples.

Example 1. Let $\mathfrak{Q}$ consist of the points $(x, y)$ of a plane, and let $B_{q_{1} q_{2} q_{2}}$ hold if the three points are on a line and $q_{2}$ is between $q_{1}$ and $q_{3}$ in the usual sense. Postulate $I$ is fulfilled. Let $\mathfrak{B}$ be the class of points in the first quadrant, and let $\mu$ be defined on $\mathfrak{P}$ so that for $p=(x, y)$ we have $\mu_{p}=y / x$, then for $l=(0,0)$ we have $\varlimsup_{\lim } \mu_{p}=0$ and $\lim \mu_{p}=+\infty$, while for every positive $a$ we have the conditions fulfilled for $\lim \mu_{p}=a$, and also we have $\lim \mu_{p}=+\infty$.

Example 2. With the same system $(\mathfrak{Q} ; B)$ and the same class $\mathfrak{B}$ as above, take $\mu$ on $\mathfrak{B}$ so that for $p=(x, y)$ we have $\mu_{p}$ equal to unity if $x=y$, equal to one half if $x$ is rational and greater than $y$, equal to zero if $x$ is irrational and greater than $y$, equal to 2 if $x$ is rational and less than $y$, and equal to 3 if $x$ 
is irrational and less than $y$. We now have a unique limit, unity, at the origin, while the upper limit is one half and the lower limit is 2.

Example 3. Again with the same system and the same class $\mathfrak{B}$, take $\mu$ on $\mathfrak{B}$ so that for $p=(x, y)$ we have $\mu_{p}$ equal to unity for $x$ rational, equal to zero for $x$ irrational and equal to or greater than $y$, and equal to 2 for $x$ irrational and less than $y$. The upper limit and the lower limit at the origin have the same value, unity, while the limit does 'not exist.

We have, however, the theorem:

TheOREM XVIII. If $\mu$ is defined on $\mathfrak{B}$, then $\mu$ can have no limit at a given limiting element $l$ of $\mathfrak{B}$ which is greater than the lower limit of $\mu$ at $l$ or less than the upper limit of $\mu$ at $l$.

As an immediate corollary we have

COROLLARY. If the upper and lower limits are equal, then if a limit exists it is unique and equal to the upper and lower limits.

For further theorems we require a second postulate, which may conveniently take the following form:

Postulate II. If $\mathfrak{S}_{1}$ and $\mathfrak{S}_{2}$ are segments containing $q$, then there is a common subclass of $\mathfrak{S}_{1}$ and $\mathfrak{S}_{2}$ which is a segment containing $q$.

This condition is clearly invariant under composition of systems, provided that the composite class contains at least one segment. Several theorems result from this postulate.

TheOREM XIX. If $\mu$ is defined on $\mathfrak{B}$, then for every limiting element $l$ of $\mathfrak{P}$ we have $\underline{\lim }_{p \rightarrow i} \mu_{p} \leq \overline{\lim }_{p \rightarrow i} \mu_{p}$.

Theorem XX. For a given $\mu$ on $\mathfrak{B}$ and a given limiting element $l$ of $\mathfrak{B}$, $a$ necessary and sufficient condition for the existence of a limit of $\mu$ at $l$ is the equality of the upper and lower limits of $\mu$ at $l$.

COROLLARY. If a limit of $\mu$ at $l$ exists, it is unique and equal to the upper and lower limits of $\mu$ at $l$.

Theorem XXI. If $\mu$ is defined on $\mathfrak{B}$ and $l$ is a limiting element of $\mathfrak{B}$, then the following two conditions are necessary and sufficient for the existence of a finite limit of $\mu$ at $l$ :

1. In every segment containing $l$ there is a $p$ distinct from $l$ such that $\mu_{p}$ is finite.

2. For every $e$ there is an $\mathfrak{S}_{0}$ containing $l$ such that, if $p_{1}$ and $p_{2}$ are in $\mathfrak{S}_{6}$ and distinct from $l$, then $\left|\mu_{p_{1}}-\mu_{p_{1}}\right| \leqq e$.

The necessity of these conditions is obvious, and the sufficiency is easily established by showing that the upper limit and the lower limit cannot be distinct.

Defrnition 11. The function $\mu$ is continuous on $\mathfrak{P}$ if $\mu_{p}$ is finite for every $p$ and if for every limiting element $l$ of $\mathfrak{B}$ that lies in $\mathfrak{B}$ we have $\lim \mu_{p} \neq \mu_{l}$.

Definition 12. The function $\mu$ is extensibly continuous on $\mathfrak{B}$ if $\mu$ is con- 
tinuous on $\mathfrak{P}$ and if for every limiting element $l$ of $\mathfrak{B}$ not in $\mathfrak{P}$ we have $\lim \mu_{p}$ existing and finite.*

TheOREM XXII. If $\mathfrak{R}$ is the derived class of $\mathfrak{B}$ and $\mu$ is defined on $\mathfrak{B}$ and $\varphi$ is defined on $\mathfrak{B}+\mathbb{Q}$ such that for every $p$ we have $\mu_{p}=\varphi_{p}$ and for every $l$ not in $\mathfrak{B}$ we have $\overparen{\lim } \mu_{p}=\varphi_{l}$, then if $\mu$ is extensibly continuous on $\mathfrak{P}, \varphi$ is extensibly continuous on $\mathfrak{B}+\mathfrak{R}$.

Since $\mathfrak{P}+\mathfrak{R}$ is closed, on this class extensible continuity is not different from ordinary continuity. For every $l$ and $e$ there exists an $\mathfrak{S}_{\text {。 }}$ containing $l$ such that for $p$ in $\Im_{e}$ we have $\left|\varphi_{p}-\varphi_{l}\right| \leqq e / 2$. For any $l_{1}$ in $\Im_{e}$ there is an $\overline{\mathfrak{S}}_{e}$ containing $l_{1}$ such that for $p$ in $\overline{\mathfrak{S}}_{e}$ we have $\left|\varphi_{l_{1}}-\varphi_{p}\right| \leqq e / 2$, and since $\widetilde{S}_{e}$ and $\overline{\mathscr{S}}_{e}$ have a common $p$, we see from these conditions that for any $q$ of $\mathfrak{B}$ or $\mathfrak{R}$ in $\mathfrak{S}_{c}$ we have $\left|\varphi_{q}-\varphi_{l}\right| \leqq e$.

\section{Functions on a composite range.}

For theorems on iterated limits we consider functions on a composite range. It is sufficient for our purpose to consider a system $(\mathfrak{Q} ; B)$, composite of two systems $\left(\mathfrak{Q}^{\prime} ; B^{\prime}\right)$ and $\left(\mathfrak{Q}^{\prime \prime} ; B^{\prime \prime}\right)$, each fulfilling Postulates I and II. The propositions of the present section relate to a function $\mu$ defined on a subclass $\mathfrak{B}$ of $\mathfrak{Q}$ of the form $\mathfrak{P}^{\prime} \mathfrak{B}^{\prime \prime}$, where $\mathfrak{P}^{\prime}$ and $\mathfrak{P}^{\prime \prime}$ are subclasses of $\mathfrak{Q}^{\prime}$ and $\mathfrak{Q}^{\prime \prime}$ respectively. The notations $l, l^{\prime}, l^{\prime \prime}$ invariably represent limiting elements respectively of $\mathfrak{B}, \mathfrak{P}^{\prime}$ and $\mathfrak{B}^{\prime \prime}$. The definite significance of these notations is presupposed in the statement of each theorem.

Theorem XXIII. If $l=l^{\prime} l^{\prime \prime}$, then

$$
\begin{aligned}
& \varliminf_{p^{\prime \prime} \rightarrow l^{\prime \prime}} \lim _{p^{\prime} \rightarrow l^{\prime}} \mu_{p^{\prime} p^{\prime \prime}} \leqq \lim _{p^{\prime \prime} \rightarrow l^{\prime \prime}} \varlimsup_{p^{\prime} \rightarrow l^{\prime}} \mu_{p^{\prime} p^{\prime \prime}} \leqq \varlimsup_{p^{\prime \prime} \rightarrow l^{\prime \prime}} \varlimsup_{p^{\prime} \rightarrow l^{\prime}} \mu_{p^{\prime} p^{\prime \prime}}, \\
& \lim _{p^{\prime \prime} \rightarrow l^{\prime \prime}} \lim _{p^{\prime} \rightarrow l^{\prime}} \mu_{p^{\prime} p^{\prime \prime}} \leqq \varlimsup_{p^{\prime \prime} \rightarrow l^{\prime \prime}} \lim _{p^{\prime} \rightarrow l^{\prime}} \mu_{p^{\prime} p^{\prime \prime}} \leqq \varlimsup_{p^{\prime \prime} \rightarrow l^{\prime \prime}} \varlimsup_{p^{\prime} \rightarrow l^{\prime}} \mu_{p^{\prime} p^{\prime \prime}}, \\
& \lim _{p \rightarrow l} \mu_{p} \leqq \lim _{p^{\prime \prime} \rightarrow l^{\prime \prime}} \lim _{p^{\prime} \rightarrow l^{\prime}} \mu_{p^{\prime} p^{\prime \prime}} \leqq \varlimsup_{p^{\prime \prime} \rightarrow l^{\prime \prime}} \varlimsup_{p^{\prime} \rightarrow l^{\prime}} \mu_{p^{\prime} p^{\prime \prime}} \leqq \varlimsup_{p \rightarrow l} \mu_{p} .
\end{aligned}
$$

(d) If $\lim \mu_{p}$ exists then

$$
\lim _{p^{\prime \prime} \rightarrow l^{\prime \prime}} \lim _{p^{\prime} \rightarrow l^{\prime}} \mu_{p^{\prime}} p^{\prime \prime}=\lim _{p^{\prime \prime} \rightarrow l^{\prime \prime}} \varlimsup_{p^{\prime} \rightarrow l^{\prime}} \mu_{p^{\prime} p^{\prime \prime}}=\lim _{p \rightarrow l} \mu_{p} .
$$

Propositions $(a)$ and $(b)$ are sufficiently obvious applications of Theorem XIX. Proposition $(d)$ is a corollary of $(c)$, which may be established as follows: We show that the assumption that the lower limit is greater than the iterated lower limit leads to a contradiction. If we make this assumption, two cases

* In the paper on Iterated Limits in General Analysis, loc. cit., we show that in many applications this condition is equivalent to uniform continuity. See especially chapter IV, theorems V, XIII, XIV and XV. 
may arise, the lower limit may be a finite number, or $+\infty$. Suppose

$$
\lim _{p \rightarrow l} \mu_{p}=a, \quad \lim _{p^{\prime \prime \rightarrow l^{\prime \prime}}} \lim _{p^{\prime} \rightarrow l^{\prime}} \mu_{p^{\prime}} p^{\prime \prime}=a-4 e .
$$

Let $\varphi$ be a function on $\mathfrak{P}^{\prime \prime}$ such that for every $p^{\prime \prime}$ we have

$$
\lim _{p^{\prime} \rightarrow l^{\prime}} \mu_{p^{\prime}} p^{\prime \prime}=\varphi_{p^{\prime \prime}} \text {. }
$$

There is an $\mathfrak{S}_{\text {c }}$ containing $l$ such that, if $p$ is distinct from $l$ in $\mathfrak{S}_{\odot}, \mu_{p} \geqq a-e$.

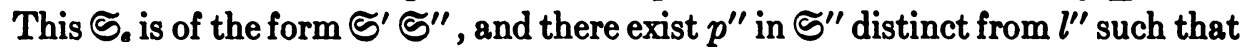
$\varphi_{p^{\prime \prime}} \leqq a-3 e$ and $p^{\prime}$ in $\mathfrak{S}^{\prime}$ distinct from $l^{\prime}$ such that $\mu_{p^{\prime}} p^{\prime \prime} \leqq \varphi_{p^{\prime \prime}}+e$. We have, therefore, $\mu_{p^{\prime}} p^{\prime \prime} \leqq a-2 e$, and the contradiction is found in the fact that $p=p^{\prime} p^{\prime \prime}$ is in $S_{\text {. }}$. If we suppose the lower limit is $+\infty$ and the iterated lower limit is finite, similar reasoning leads to a contradiction.

Theorem XXIV. (a) If $l^{\prime}$ is such that for every $l$ of the form $l=l^{\prime} p^{\prime \prime}$ we have

$$
\lim _{p \rightarrow l} \mu_{p}=\varphi_{p}^{\prime \prime},
$$

where $\varphi$ is a finite valued function on $\mathfrak{B}^{\prime \prime}$, then $\varphi$ is continuous on $\mathfrak{P}^{\prime \prime}$.

(b) If $l^{\prime}$ is such that for every $l$ of the form $l=l^{\prime} q^{\prime \prime}, q^{\prime \prime}$ being in $\mathfrak{B}^{\prime \prime}+\mathbb{R}^{\prime \prime}$, we have

$$
\lim _{p \rightarrow l} \mu_{p}=\varphi_{q^{\prime \prime}},
$$

where $\varphi$ is a finite valued function on $\mathfrak{B}^{\prime \prime}+\mathfrak{R}^{\prime \prime}$, then $\varphi$ is extensibly continuous on $\mathfrak{B}^{\prime \prime}+\mathfrak{R}^{\prime \prime}$.

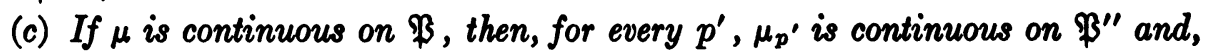
for every $p^{\prime \prime}, \mu_{p}$ " is continuous on $\mathfrak{B}^{\prime}$.*

(d) If $\mu$ is extensibly continuous on $\mathfrak{B}$, then, for every $p^{\prime}, \mu_{p^{\prime}}$ is extenoibly continuous on $\mathfrak{P}^{\prime \prime}$ and, for every $p^{\prime \prime}, \mu_{p^{\prime \prime}}$ is extensibly continuous on $\mathfrak{B}^{\prime}$.

These propositions may be regarded as corollaries of those of the theorem just preceding.

In the remaining theorems of this section we employ notions analogous to that of uniform convergence of a sequence of functions, and to that of a family of equally continuous functions. $f$

Defintrion 13. If $\varphi$ is defined and finite on $\Re^{\prime \prime}$, a subclass of $\mathfrak{P}^{\prime \prime}, \mu_{p^{\prime}}$ is said to approach $\varphi$ uniformly on $\Re^{\prime \prime}$ as $p^{\prime}$ approaches $l^{\prime}$ if for every $e$ there exists an $\mathfrak{S}^{\prime}$. containing $l^{\prime}$ such that if $p^{\prime}$ is in $\mathfrak{S}^{\prime}$. distinct from $l^{\prime}$ and $p^{\prime \prime}$ is in $\Re^{\prime \prime}$ we have $\left|\mu_{p^{\prime}} p^{\prime \prime}-\varphi_{p^{\prime \prime}}\right| \leqq e$. The notation for this condition is $\lim _{p^{\prime} \rightarrow l^{\prime}} \mu_{p^{\prime}}=\varphi\left(\Re^{\prime \prime}\right)$.

"The notation $\mu_{p^{\prime}}$ indicates a function on $\mathfrak{P}^{\prime \prime}$, the argument $p^{\prime}$ being fixed while $p^{\prime \prime}$ rangee over the class \$" $^{\prime \prime}$.

† Fréchet, loc. cit., p. 10. 
DeFnnimion 14. The function $\mu$ is continuous on $\mathfrak{P}^{\prime}$ uniformly on $\mathfrak{P}^{\prime \prime}$ if for every $l^{\prime}$ in $\mathfrak{B}^{\prime}$ we have $\lim _{\boldsymbol{\nu} \rightarrow \nu} \mu_{p^{\prime}}=\mu_{l^{\prime}}\left(\mathfrak{P}^{\prime \prime}\right)$.

Definimion 15. The function $\mu$ is extensibly continuous on $\mathfrak{B}^{\prime}$ uniformly on $\mathfrak{P}^{\prime \prime}$ if it is continuous on $\mathfrak{P}^{\prime}$ uniformly on $\mathfrak{B}^{\prime \prime}$ and if for every $l^{\prime}$ not in $\mathfrak{P}^{\prime}$ there is a finite valued function $\varphi$ on $\mathfrak{P}^{\prime \prime}$ such that we have $\lim _{p^{\prime} \rightarrow l^{\prime}} \mu_{p^{\prime}}=\varphi\left(\mathfrak{P}^{\prime \prime}\right)$.

Theorem XXV. If $l=l^{\prime} l^{\prime \prime}$ and $\Re^{\prime \prime}$ is the class of all elements $p^{\prime \prime}$ in some fixed $\mathfrak{S}^{\prime \prime}$ containing $l^{\prime \prime}$, then if $\varphi$ is a finite valued function on $\mathfrak{B}^{\prime \prime}$ such that

we have

$$
\lim _{p^{\prime} \rightarrow l^{\prime}} \mu_{p^{\prime}}=\varphi\left(\Re^{\prime \prime}\right)
$$

$$
\lim _{p \rightarrow l} \mu_{p}=\lim _{p^{\prime \rightarrow} \rightarrow \nu^{\prime}} \varphi_{p^{\prime \prime}}, \quad \varlimsup_{p \rightarrow l} \mu_{p}=\varlimsup_{p^{\prime \prime \rightarrow l^{\prime \prime}}} \varphi_{p^{\prime \prime}},
$$

provided that in case $l^{\prime}$ is in $\mathfrak{B}^{\prime}$ we have the additional hypothesis

$$
\lim _{p^{\prime \prime \rightarrow l^{\prime \prime}}} \varphi_{p^{\prime \prime}} \leqq \lim _{p^{\prime \prime \rightarrow l^{\prime \prime}}} \mu_{l^{\prime}} p^{\prime \prime}, \quad \varlimsup_{p^{\prime \prime} \rightarrow l^{\prime \prime}} \mu_{l^{\prime}} p^{\prime \prime} \leqq \varlimsup_{p^{\prime \prime} \rightarrow l^{\prime \prime}} \varphi_{p^{\prime \prime}},
$$

and in case $l^{\prime \prime}$ is in $\Re^{\prime \prime}$ the hypothesis

$$
\lim _{p^{\prime \prime \rightarrow} \rightarrow{ }^{\prime \prime}} \varphi_{p^{\prime \prime}} \leqq \varphi_{l^{\prime \prime}} \leqq \varlimsup_{p^{\prime \prime \rightarrow} \rightarrow{ }^{\prime \prime}} \varphi_{p^{\prime \prime}} . *
$$

Denote the lower limits in (1) by $L$ and $L^{\prime \prime}$. We prove $L=L^{\prime \prime}$ for the case when $l^{\prime}$ is not in $\mathfrak{P}^{\prime}$ and $l^{\prime \prime}$ is not in $\mathfrak{P}^{\prime \prime}$. That $L$ is not greater than $L^{\prime \prime}$ follows from Theorem XXIII, (c). Now suppose that $L^{\prime \prime}$ is the greater. Consider first the case when $L^{\prime \prime}=a$, and let $L=a-4 e$. There exists an $\mathfrak{S}^{\prime \prime}$ containing $l^{\prime \prime}$ and contained in the fixed $\mathfrak{S}^{\prime \prime}$ such that if $p^{\prime \prime}$ is in $\mathfrak{S}^{\prime}$. we have $\varphi_{p^{\prime \prime}} \geqq a-e$, and there exists $\mathfrak{S}^{\prime}$. containing $l^{\prime}$ such that if $p^{\prime}$ is in $S^{\prime}$, and $p^{\prime \prime}$ in $\mathcal{S}^{\prime \prime}$, we have $\left|\mu_{p^{\prime}} p^{\prime \prime}-\varphi_{p^{\prime \prime}}\right| \leqq e$, whence $\mu_{p^{\prime}} p^{\prime \prime} \geqq a-2 e$. Also, in every $\subseteq$ containing $l$ there is a $p$ such that $\mu_{p} \leqq a-3 e$. We find a contradiction when $\mathfrak{S}=\mathfrak{S}^{\prime} \mathfrak{S}^{\prime \prime}$. Consider now $L^{\prime \prime}=+\infty$ and $L=\underline{a}$. For a given $a$ there is an $\mathfrak{S}_{a}^{\prime \prime}$ containing $l^{\prime \prime}$ and contained in $\mathfrak{S}^{\prime \prime}$ such that if

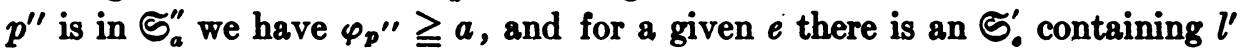
such that if $p^{\prime}$ is in $\mathfrak{S}_{\text {, and }}^{\prime} p^{\prime \prime}$ is in $\widetilde{S}_{a}^{\prime \prime}$ we have $\left|\mu_{p^{\prime}} p^{\prime \prime}-\varphi_{p^{\prime \prime}}\right| \leqq e$, whence $\mu_{p^{\prime}} p^{\prime \prime} \geqq a-e$. Also, in every $\mathfrak{S}$ containing $l$ there is a $p$ such that $\mu_{p} \leqq \underline{a}+e$. We find a contradiction when $\mathfrak{S}=\mathfrak{S}_{e}^{\prime} \mathfrak{S}_{a}^{\prime \prime}$ and $a>\underline{a}+2 e$.

The equality of the upper limits for this case follows by analogy, and in case the restriction as to $l$ is removed the additional hypotheses then provided clearly validate the conclusions.

Similar considerations lead also to the following theorem, which gives similar results from milder hypotheses.

- We omit the corresponding propositions involving uniform approach to $t \infty$ and to $-\infty$, which are stated in the American Journal of Mathematics (loc. cit., chapter III, theorem $\mathrm{X}$ ), and are valid here. 
Theorem XXVI. If $l=l^{\prime} l^{\prime \prime}$ and $l^{\prime \prime}$ is not in $\mathfrak{P}^{\prime \prime}$, and if $\varphi$ and $\theta$ are finite valued functions on $\mathfrak{F}^{\prime \prime}$, then

(a) $A$ sufficient condition for

$$
\varlimsup_{p \rightarrow l} \mu_{p} \leqq \varlimsup_{p^{\prime \prime} \rightarrow l^{\prime \prime}} \varphi_{p^{\prime \prime}}
$$

is that there exist an S" $^{\prime \prime}$ containing $l^{\prime \prime}$ such that for every e there is an $\mathbb{S}^{\prime}$. containing $l^{\prime}$ such that if $p^{\prime}$ is in $\mathcal{S}^{\prime}$, and $p^{\prime \prime}$ is in $\mathcal{S}^{\prime \prime}$ then $\mu_{p^{\prime}} p^{\prime \prime} \leqq \varphi_{p^{\prime \prime}}+e$ :

(b) A sufficient condition for

$$
\lim _{p \rightarrow l} \mu_{p} \geqq \lim _{, x^{\prime \prime} \rightarrow x^{\prime \prime}} \theta_{p^{\prime \prime}}
$$

is that there exist an $\mathfrak{S}^{\prime \prime}$ containing $l^{\prime \prime}$ such that for every e there is an $\mathfrak{S}_{\text {, containing }}^{\prime}$ $l^{\prime}$ such that if $p^{\prime}$ is in $\mathcal{S}^{\prime}$, and $p^{\prime \prime}$ is in $\mathcal{S}^{\prime \prime}$ then $\mu_{p^{\prime}} p^{\prime \prime} \geqq \theta_{p^{\prime \prime}}-e$.

Theorem XXVII. If $l=l^{\prime} l^{\prime \prime}$ and $S^{\prime \prime}$ contains $l^{\prime \prime}$ and $\Re^{\prime \prime}$ consists of all elements $p^{\prime \prime}$ in $\mathfrak{S}^{\prime \prime}$, and if in every $\mathfrak{S}^{\prime}$ containing $l^{\prime}$ there is a $p^{\prime}$ distinct from $l^{\prime}$ such that, for some $a$,

$$
\lim _{p^{\prime \prime} \rightarrow x^{\prime \prime \prime}} \mu_{p^{\prime}} p^{\prime \prime}=a,
$$

and if $\varphi$ is a finite valued function on $\mathfrak{B}^{\prime \prime}$ such that

$$
\lim _{p^{\prime} \rightarrow \nu^{\prime}} \mu_{p^{\prime}}=\varphi\left(\Re^{\prime \prime}\right),
$$

then there is an $a_{1}$ such that

$$
\lim _{p^{\prime \prime} \rightarrow x^{\prime \prime}} \varphi_{p^{\prime \prime}}=a_{1} \text {. }
$$

For by the uniform approach to $\varphi$ we have for every $e$ an $\mathcal{S}^{\prime}$, containing $l^{\prime}$ such that if $p^{\prime}$ is in $\mathfrak{S}^{\prime}$ distinct from $l^{\prime}$ and if $p^{\prime \prime}$ is in $\mathcal{S}^{\prime \prime}$ then

$$
\left|\mu_{p^{\prime}} p^{\prime \prime}-\varphi_{p^{\prime \prime}}\right| \leqq e / 3 \text {. }
$$

Also there is a $p_{1}^{\prime}$ in $\widetilde{S}_{\text {。 }}^{\prime}$ such that, taking account of Theorem XXI, there exists an $\mathfrak{S}_{1}^{\prime \prime}$ containing $l^{\prime \prime}$ such that if $p_{1}^{\prime \prime}$ and $p_{2}^{\prime \prime}$ are in $\mathfrak{S}_{1}^{\prime \prime}$ and distinct from $l^{\prime \prime}$ we have

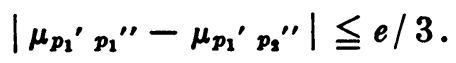

Take $\mathfrak{S}_{\text {. }}^{\prime \prime}$ common to $\mathfrak{S}^{\prime \prime}$ and $\mathfrak{S}_{1}^{\prime \prime}$, then if $p_{1}^{\prime \prime}$ and $p_{2}^{\prime \prime}$ are in $\mathfrak{S}_{0}^{\prime \prime}$ we have from (1) and (2) the condition $\left|\varphi_{p_{1}}{ }^{\prime \prime}-\varphi_{p_{2}}{ }^{\prime \prime}\right| \leqq e$, and since condition (1) of Theorem XXI is obviously fulfilled by $\varphi$ at $l^{\prime \prime}$, we have the desired conclusion.

The two following theorems are easily deduced from the preceding. The propositions are true either with or without the words in brackets.

Theorem XXVIII. If $l^{\prime}$ is such that in every $\mathfrak{S}^{\prime}$ containing $l^{\prime}$ there is a $p^{\prime}$ distinct from $l^{\prime}$ such that $\mu_{p^{\prime}}$ is [extensibly] continuous on $\mathfrak{P}^{\prime \prime}$, and if $\varphi$ is a 
finite valued function on $\mathfrak{P}^{\prime \prime}$ such that

$$
\lim _{p^{\prime} \rightarrow l^{\prime}} \mu_{p^{\prime}}=\varphi\left(\mathfrak{P}^{\prime \prime}\right),
$$

then $\varphi$ is [extensibly] continuous on $\mathfrak{P}^{\prime \prime}$.

TheOREM XXIX. If $\mu$ is [extensibly] continuous on $\mathfrak{P}^{\prime}$ uniformly on $\mathfrak{P}^{\prime \prime}$, and if for every $p^{\prime} \mu_{p^{\prime}}$ is [extensibly] continuous on $\mathfrak{P}^{\prime \prime}$, then $\mu$ is [extensibly] continuous on $\mathfrak{P}$.

\section{Theorems requiring a third postulate.}

Consider a class $\mathfrak{Q}$ consisting of all integers, positive and negative. Let $B_{q_{1} q_{2} q_{8}}$ hold if $q_{1}$ and $q_{3}$ are integral multiples of 4 and $q_{2}$ is between them in the usual sense. The system $(\mathfrak{O} ; B)$ fulfils Postulates I and II, but clearly violates several conditions usually considered as fundamental in the theory of limits. For example, the proposition that no finite class has a limiting element does not hold, since any class containing two successive integers has one of these as a limiting element. Again, the proposition that a sequence can have but one limit does not hold, for the sequence $2,2,2 \ldots$ has the limits 1 , 2 , and 3 . These conditions and certain others that may be desirable in extending a theory of limits are fulfilled in a system $(\mathfrak{O} ; B)$ restricted by the following postulate.

Postulate III. If $q_{1}$ and $q_{2}$ are distinct elements, each contained in some segment, then there exist segments, $\mathfrak{\Im}_{1}$ containing $q_{1}$ and $\mathfrak{\Im}_{2}$ containing $q_{2}$, such that $\mathfrak{S}_{1}$ and $\mathfrak{S}_{2}$ contain no common element.

This postulate, like the first two, is invariant under composition of systems if we exclude the trivial case in which the composite class contains no segment. Also it is obviously fulfilled by any simply ordered class, if the relation $B$ plays the rôle of betweenness.

On the basis of the three postulates our definition of limiting element is found to fulfil the four conditions regarded as fundamental by F. Riesz.* The first is expressed in Theorem III, and the remaining conditions are embodied in the propositions of the following theorem.

Theorem XXX. (a) If $\mathfrak{P}=\mathfrak{P}_{1}+\mathfrak{B}_{2}$ then every limiting element of $\mathfrak{B}$ is a limiting element of $\mathfrak{B}_{1}$ or of $\mathfrak{B}_{2}$.

(b) No finite class has a limiting element.

(c) Each limiting element of a class $\mathfrak{B}$ is uniquely determined by the totality of all subclasses of $\mathfrak{B}$ of which it is a limiting element.

Proposition (a) is seen to depend only on Postulate II. As to (b), if $\mathfrak{B}$ consists of a finite set, $p_{1}, p_{2}, \cdots, p_{n}$, then for any $q$ there exist, by Postulates I and III, segments $\mathfrak{S}_{1}, \mathfrak{S}_{2}, \cdots, \mathfrak{S}_{n}$ containing $q$ such that if $p_{i} \neq q$ the seg-

* Loc. cit. 
ment $\mathfrak{S}_{i}$ does not contain $p_{i}$. By Postulate II we secure a segment $\mathfrak{S}$ contained in all the $\mathfrak{S}_{i}$ but containing $q$. Since $\mathfrak{S}$ does not contain any $p$ distinct from $q, q$ is not a limiting element of $\mathfrak{B}$. To prove (c), let $q_{1}$ and $q_{2}$ be two distinct elements, then by Postulates I and III there exist $\Im_{1}$ containing $q_{1}$ and $\mathfrak{S}_{2}$ contoining $q_{2}$ such that $\mathfrak{S}_{1}$ and $\mathfrak{S}_{2}$ have no common elements. If $q_{1}$ and $q_{2}$ are limiting elements of $\mathfrak{B}$, they are limiting elements respectively of the two subclasses $\mathfrak{P}-\mathfrak{S}_{2}$ and $\mathfrak{P}-\mathfrak{S}_{1}$, but $q_{1}$ is not a limiting element of $\mathfrak{B}-\mathfrak{S}_{1}$, nor $q_{2}$ of $\mathfrak{B}-\widetilde{\subseteq}_{2}$.

Theorem XXXI. A sequence cannot have two distinct limits.

Theorems VI, VII and XXXI show that our definition of limit of a sequence fulfils the conditions used by $M$. Fréchet on page 5 of his thesis. We can now prove the two following theorems, the first stated after the manner of a theorem by Fréchet, the second after a theorem by Riesz.

TheOREM XXXII. If every member of a sequence of subclasses of a compact class is closed, contains the succeeding member and contains at least one element, then there is an element common to all members of the sequence.

Theorem XXXIII. If every member of a sequence of infinite subclasses of a compact class contains the succeeding member, the members of the sequence have a common limiting element.

To prove this last theorem let $\left\{\mathfrak{P}_{n}\right\}$ be such a sequence of subclasses and let $\left\{q_{n}\right\}$ be a sequence of distinct elements such that, for every $n, q_{n}$ is in $\mathfrak{P}_{n}$. The class $\mathfrak{P}$ of all elements in the sequence $\left\{q_{n}\right\}$ is infinite and has at least one limiting element $q$. This $q$ is a limiting element of every class of the sequence $\left\{\mathfrak{B}_{n}\right\}$, by Theorem XXX; for, any given member of the sequence of classes contains all but a finite number of elements of the sequence of elements.

Theorem XXXII, which does not follow from the work of Frechet because of the difference in definitions of limiting elements, is an immediate corollary of the theorem just proved.

The three postulates are sufficient also to secure the following form of the Heine-Borel theorem.

THEOREM XXXIV. If $\left\{\mathfrak{P}_{n}\right\}$ is a sequence of subclasses of $\mathfrak{Q}$ such that every element of a certain class $\mathfrak{B}$ which is compact and closed is interior to at least one of the $\mathfrak{B}_{i}$, then there is a finite set of the $\mathfrak{B}_{i}$ such that every element of $\mathfrak{B}$ is interior to at least one class of the finite set.

Suppose the theorem is untrue. Then there exists a sequence $\left\{p_{m}\right\}$ of distinct elements of $\mathfrak{B}$ such that $p_{m}$ is not interior to $\mathfrak{B}_{n}$ if $n<m$. The class $\overline{\mathfrak{P}}$ of all elements of the sequence $\left\{p_{m}\right\}$ has a limiting element $p$, which is in $\mathfrak{P}$ and therefore interior to some $\mathfrak{P}_{i}$, say $\mathfrak{B}_{n_{1}}$. Let $\overline{\mathfrak{P}}=\mathfrak{B}_{1}+\mathfrak{B}_{2}$, where $\mathfrak{P}_{1}$ is the part interior to $\mathfrak{P}_{n_{1}}$, and $\mathfrak{P}_{2}$ is the remainder. $\mathfrak{P}_{1}$ is finite and has no limiting element. There is a segment $\mathfrak{S}$ containing $p$ and contained in $\mathfrak{B}_{n_{1}}$, 
therefore containing no element of $\mathfrak{B}_{2}$, so that $p$ is not a limiting element of $\mathfrak{P}_{2}$, and consequently not of $\overline{\mathfrak{P}}$. Our supposition is therefore false.

The proof of the following theorem, which, in view of Theorem $\operatorname{XXX}(a)$, implies Theorem I, is obvious.

TheOREM XXXV. The derived class of any class is closed.

To the list of theorems rendered valid by the addition of the third postulate may be added the following propositions on functions.

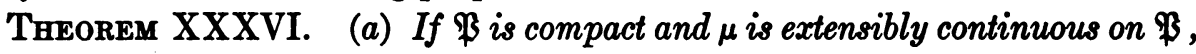
then $\mu$ is finitely bounded on $\mathfrak{B}$.

(b) If $\mathfrak{B}$ is compact, then there exist $q_{1}$ and $q_{2}$ such that the the least upper bound of $\mu$ on $\mathfrak{B}$ is either $\mu_{q_{1}}$ or $\varlimsup_{p \rightarrow q_{1}} \mu_{p}$, and the greatest lower bound of $\mu$ on $\mathfrak{P}$ is either $\mu_{q}$ or $\lim _{p \rightarrow q_{q}} \mu_{p}$.

(c) If $\mathfrak{B}$ is compact and closed and $\mu$ is continuous on $\mathfrak{B}$, then there exist $p_{1}$ and $p_{2}$ such that the least upper bound of $\mu$ on $\Re$ is $\mu_{p_{1}}$ and the greatest lower bound of $\mu$ on $\mathfrak{B}$ is $\mu_{p_{s}}$.

Proof of $(a)$. Suppose that $\mu$ is not bounded from $+\infty$, then it can easily be shown that there exists a sequence $\left\{p_{n}\right\}$ of distinct elements such that

$$
\lim _{n \rightarrow \infty} \mu_{p_{n}}=+\infty \text {. }
$$

The elements of this sequence constitute a class having a limiting element, say $l$. Since $\mu$ is extensibly continuous, there is a segment $\subseteq$ containing $l$ such that for some $a$ and for every $p$ in $\subseteq$ distinct from $l$ we have $\mu_{p}<a$. Since $\subseteq$ must contain an infinite number of elements of the sequence $\left\{p_{n}\right\}$, we reach a contradiction. Similarly it may be shown that $\mu$ is bounded from $-\infty$.

Proof of $(b)$. We consider the cases when the least upper bound of $\mu$ on $\mathfrak{P}$ is finite and $+\infty$. In either case there exists an element $p$ such that $\mu_{p}$ is this least upper bound, or there is a sequence $\left\{p_{n}\right\}$ of distinct elements such that the limit of $\mu_{p_{\mathrm{s}}}$ is this least upper bound. If the latter situation prevails, then this sequence gives rise to a limiting element $l$, and the assumption that the upper limit of $\mu$ at $l$ is different from the least upper bound leads obviously to a contradiction. Thus if not a $p$, then an $l$ will serve as the $q_{1}$ required. Similarly we may show the existence of a $q_{2}$ meeting the requirements of the theorem.

Proposition (c) is a corollary of $(b)$.

TrEorem XXXVII. If $\mu$ is finitely bounded on $\$$, then, for a fixed positive $a$, the set of elements $l$ at which

is closed.

$$
\varlimsup_{p \rightarrow l} \mu_{p}-\varlimsup_{p \rightarrow l} \mu_{p} \geqq a
$$


Suppose every segment containing $l$ contains an $l_{1}$ for which the upper and lower limits of $\mu$ differ by a quantity greater than or equal to $a$. Let the upper limit and lower limit at $l$ be respectively $\bar{a}$ and $\underline{a}$, and suppose that $\bar{a}-\underline{a}<a$. Take $e$ as one sixth of $a-\bar{a}+\underline{a}$, then there is an $\mathcal{S}_{b}$ containing $l$ such that if $p$ is in $\mathfrak{S}_{e}$ and distinct from $\bar{l}$ we have $\underline{a}-e \leqq \mu_{p} \leqq \bar{a}+e$, and therefore if $p_{1}$ and $p_{2}$ are in $\varsigma_{6}$ and distinct from $l$ we have $\left|\mu_{p_{1}}-\mu_{p_{2}}\right|$ $\leqq a-4 e$. But if $l_{1}$ is in $\mathfrak{S}_{3}$ and such that the upper limit and lower limits of $\mu$ at $l_{1}$ are respectively $\bar{a}_{1}$ and $\underline{a}_{1}$, where $\bar{a}_{1}-\underline{a}_{1} \geqq a$, then we may take $p_{1}$ and $p_{2}$ in $\widetilde{S}_{e}$ distinct from $l$ such that $\mu_{p_{1}} \geqq \bar{a}_{1}-e$ and $\mu_{p_{8}} \leqq \underline{a}_{1}+e$, whence $\left|\mu_{p_{1}}-\mu_{p_{2}}\right| \geqq a-2 e$, and this affords a contradiction.

\section{A sequential theory for a $\operatorname{system}(\mathfrak{Q} ; B)$.}

In the theory of point sets a limiting point of a set may be defined as a point every neighborhood of which contains a point of the set distinct from itself, or as a point which is the limit of a sequence of distinct points of the set. The notion " limit of a sequence " being defined in terms of " neighborhood," the two definitions are known to be equivalent. In the domain of general analysis, where elements of arbitrary character are considered, it may happen that, even though the notions involved in both definitions of limiting element are well defined, and even though either definition may lead to a theory of very considerable extent, the two theories need not be equivalent.

The definition of limiting element we have employed in the foregoing sections is an instance of a " neighborhood" definition, and the theory developed is in terms of " segment," which is an instance of the general notion of neighborhood. The theory might be characterized as a " neighborhood theory" for a system $(\mathfrak{Q} ; B)$. But since the notion "limit of a sequence" is defined (Def. 7), we might adopt a " sequential " definition of limiting element and develop a "sequential theory" for a system of this type. Fortunately, however, this theory becomes a special case of a general theory receiving consideration in papers by M. Fréchet,* E. R. Hedrick, † and T. H. Hildebrandt. Our definition of limit of a sequence satisfies all conditions used by Fréchet and Hildebrandt in the purely sequential parts of their papers. Hildebrandt shows that for many of the theorems none of these conditions are necessary. It is obviously true, also, that in this sequential theory we should have the theorem " every derived class is closed," which in the neighborhood theory must be stated, " if every element of a sequence whose limit is $q$ is a limit of a

\footnotetext{
"The first part of the paper by M. Fréchet referred to above is purely "sequential" in character.

†On Properties of a Domain for which any Derived Set is Closed, these T r a $\mathrm{n}$ a c $\mathrm{t}$ i o $\mathrm{n}$, vol. 12 (1911), pp. 285-294.

† A Contribution to the Foundations of Frechet's Calcul Fonctionnel, A m e r i c a n J o u r n a l of Mathematic s, vol. 34 (1912), p. 237.
} 
sequence of distinct elements of $\mathfrak{B}, q$ is a limit of a sequence of distinct elements of $\mathfrak{B}$." This proposition, with the conditions on "limit" postulated by Fréchet, forms the basis for the first part of the paper by Hedrick.

It appears, then, that a considerable body of " sequential theory" is available for a system $(\mathfrak{Q} ; B)$ subject to our three postulates. We show by the following two examples that for such a system theorems of like form in the sequential and neighborhood theories need not be equivalent theorems.

ExAmple 4. Let $\mathfrak{O}$ be the class of all infinite sequences of real numbers. For any three such sequences, $q_{1}=q_{1}^{1}, q_{1}^{2}, q_{1}^{3}, \cdots, q_{2}=q_{2}^{1}, q_{2}^{2}, q_{2}^{3}, \cdots ;$ and $q_{3}=q_{3}^{1}, q_{3}^{2}, q_{3}^{3}, \cdots$, let the relation $B_{q_{1} q_{3} q_{8}}$, hold if and only if, for every value of $i$, we have either $q_{1}^{i}<q_{2}^{i}<q_{3}^{i}$ or $q_{3}^{i}<q_{2}^{i}<q_{1}^{i}$. The three postulates are obviously fulfilled by this system $(\mathfrak{Q} ; B)$. Let $\mathfrak{B}$ be the subclass consisting of all infinite sequences of positive real numbers. The element $q=0,0,0$, ... is a limiting element of $\mathfrak{B}$, but $q$ is not the limit of any sequence of elements of $\mathfrak{B}$. For suppose the sequence $\left\{p_{n}\right\}$ to have the limit $q$, then the element $p=p^{1}, p^{2}, p^{3}, \cdots$, where $p^{i}=p_{i}^{i}$, with any sequence of negative real numbers, clearly determines a segment such that no element of the sequence $\left\{p_{n}\right\}$ is in the segment.

The system $(\mathfrak{Q} ; B)$ of this example may be regarded as a composite system, composite of a denumerable infinitude of systems $\left(\mathfrak{Q}^{i} ; B^{i}\right)$, in each of which $\mathfrak{D}^{i}$ is the class of all real numbers and $B^{i}$ is the ordinary relation of betweenness.

Example 5. Let $\Re$ be a non-denumerable class of elements $k$ to which a definite normal order has been assigned, i. e., let $\Omega$ be well-ordered but not capable of a one-to-one correspondence with the class of natural numbers or a subclass of it.* Let $\mathfrak{B}$ consist of all denumerable subclasses of $\mathfrak{\Omega}$, and let $\mathfrak{Q}$ consist of all elements of $\mathfrak{P}$ together with all real numbers except those between zero and one. For this class $\mathfrak{Q}$ we define first a relation of precedence $(<)$. With respect to the real numbers in $\mathfrak{Q}$ the relation precedes shall have the usual significance. Every element $p$ of $\mathfrak{B}$ shall precede all positive real numbers in $\mathfrak{Q}$, and shall follow all other real numbers in $\mathfrak{Q}$. Of two distinct elements, $p_{1}$ and $p_{2}$, of $\mathfrak{B}$, that one precedes which contains the first element $k$, according to the normal order of $\Omega$, which is in one and only one of the two subclasses $p_{1}$ and $p_{2}$ of $\Omega$. The relation of precedence so defined is transitive, 80 that the conditions for linear order are fulfilled. It may also be shown that $\mathfrak{Q}$ is dense, and that the pseudo-archimedean postulate used by Veblen is fulfilled. $\dagger$

Let $B_{q_{1} q_{3} q_{2}}$ hold if and only if $q_{1}<q_{2}<q_{8}$ or $q_{8}<q_{2}<q_{1}$, then it is clear that all three of our postulates are fulfilled by the system $(\mathfrak{Q} ; B)$.

- Compare O. Veblen, Definition in Terms of Order Alone in the Linear Continuum and in Well-ordered Sets, these Tra n a c t i o n s, vol. 6 (1905), p. 169.

† O. Veblen, loc. cit., p. 165. 
Now the element $q_{0}=0$ is a limiting element of the subclass $\mathfrak{B}$. For, given any $p$, a denumerable subclass of $\Re$, we may take $p_{1}$ as a subclass of $\Omega$ including every $k$ in $p$ and one additional $k$; then $p_{1}<p$. But no sequence $\left\{p_{n}\right\}$ of elements of $\mathfrak{B}$ can have the limit $q_{0}$; for the least common superclass of the subclasses $p_{i}$ that occur in the sequence is denumerable, and an element $p$ consisting of this and one $k$ in addition will precede every $p_{i}$.

It thus appears that the equivalence of the "neighborhood" and the " sequential" definitions of limiting element involves serious restrictions on a system $(\mathfrak{Q} ; B)$, in addition to our postulates, and even in addition to the assumption that $\mathfrak{Q}$ is simply ordered, dense, and satisfies the pseudo-archimedean postulate.

\section{The sequential property.}

We shall say that a subclass $\mathfrak{B}$ of $\mathfrak{Q}$ has the sequential property if every subclass of $\mathfrak{B}$ that has a limiting element $q$ contains a sequence of distinct elements whose limit is $q$. This property is definite for any subclass of a class $\mathfrak{Q}$ for which the two notions, "limiting element of a subclass" and "limit of a sequence" are defined.* In particular, if the idea of neighborhood of an element is available for a class $\mathfrak{Q}$, then definitions of the form of definitions 2 and 7 of section 2 afford a definite sequential property.

Relative to a system of the type $(\mathfrak{D} ; B)$ we consider the following postulates.

Postulate IV. For every $q$ there exists a sequence $\left\{\mathfrak{S}_{n}\right\}$ of segments containing $q$ such that, for every segment $\Im$ containing $q$, there is an $n_{\odot}$ such that, if $n>n_{\varpi}, \mathfrak{S}_{n}$ is a subclass of $\widetilde{\Xi}$.

Postulate $I V^{\prime}$. For every $q$ there exists a sequence $\left\{\mathfrak{S}_{n}\right\}$ of segments containing $q$ and having no other common element.

Each of these postulates is invariant under composition, provided only that the composite system shall possess at least one element. If $\mathfrak{Q}$ is simply ordered and $B$ is the ordinary relation of betweenness, then these two postulates are equivalent, and each is equivalent to the assumption that $\mathfrak{Q}$ has the sequential property. Without the assumption of linear order, it is easily seen that Postulate IV implies I and II, and that III and IV together imply IV'. The illustration at the beginning of section 7 is an instance of a system fulfilling I, II and IV, but not III or IV'. Example 4 fulfils I, II, III and IV' but not IV. It may be shown that in any system fulfilling Postulates III and IV the class $\mathfrak{Q}$ has the sequential property, and therefore that for such a system the sequential theory and the neighborhood theory are equivalent.

Systems which satisfy Postulates III and IV, and therefore I, II and IV', are of very frequent occurrence. In the definition of the linear continuum

* The question of a sequential property is obviously trivial in case the sequential definition of limiting element is employed. It is conceivable that various definitioris of limiting element may be given, without the use of sequence or neighborhood. 
given by $O$. Veblen, condition $A$ (linear order) and condition $E$ (postulate of uniformity), the latter so modified as to be effective without $B, C$, and $D$, and weakened by the omission of properties 1 and 3 , are sufficient to secure such a system, where the relation $B$ is ordinary betweenness." This modified $E$ may be replaced by the stronger "postulate of linearity" which is due to G. Cantor and occurs in E. V. Huntington's exposition of the modern theory of the continuum. $\dagger$ This postulate would require that there exist a denumerable subclass $\Re$ of $\mathfrak{Q}$ such that every segment must contain at least one element of $\Re$, and that $\mathfrak{Q}$ shall be dense.

Turning now to a consideration of the sequential property in relation to a general notion of " neighborhood," of which the notion segment is obviously a special case, we observe that this property is present in systems of much more general character than a system $(\mathfrak{Q} ; B)$ fulfilling Postulates III and IV. In the paper on iterated limits in general analysisł we take the notion "neighborhood" as undefined, that is, a relation $T$ between subclass and element is undefined but subject to postulates that validate a theory in which a class having the relation $T$ to an element serves as a neighborhood of that element. The postulates, stated in the second chapter of the paper, pertain to a system $(\mathfrak{B} ; \mathfrak{u} ; T)$, in which $\mathfrak{B}$ is a class of elements, $\mathfrak{u}$ is a class of ideal elements, and $T$ is a relation between subclasses of $\mathfrak{P}$ and elements of the class $\mathscr{Q}=\mathfrak{B}$ $+\mathfrak{u}$. We obtain a special instance of a system of this type from a system $(\mathfrak{Q} ; B)$ if we set $\mathfrak{B}=\mathfrak{Q}$, let $\mathfrak{u}$ be the null class, and let a class $\Re$ have the relation $T$ to an element $p$ if and only if $\Re$ is a segment containing $p$. Postulates III and IV on the system $(\mathscr{D} ; B)$ are sufficient to secure the five postulates used for the system $(\mathfrak{B} ; \mathfrak{u} ; T)$, in fact, all but the third and fifth result from the type of $(\mathfrak{Q} ; B)$, without the use of postulates. Now Theorem IV of the second chapter of the paper cited shows that every subclass has the sequential property; and the proof of this theorem involves only Postulates (I), (II), (III) and (V), while (II) may be dispensed with in case no ideal elements are present.

Other systems in terms of which a system $(\mathfrak{B} ; \mathfrak{u} ; T)$ may be set up in such manner as to satisfy Postulates (I)-(V), and which, therefore, secure the sequential property, are discussed in the fourth chapter of the same paper. These include cases of neighborhood defined in terms of voisinage as used by $M$. Fréchet, $\delta$ and in terms of suitably conditioned $K$ relations of the type used by E. H. Moore $\|$ and T. H. Hildebrandt. T

O. Veblen, loc. cit., p. 166.

† E. V. Huntington, A n a ls of M a the mati c s, ser. 2, vol. 7 (1905), p. 15.

$\$$ Loc. cit.

SRendicontidel Circolo Matematico di Palermo, vol. 22 (1906), p. 17.

|l Introduction to a Form of General Analysis, part II, p. 125 et seq.

I Loc. cit., p. 242. 
In his paper on properties of a domain for which any derived set is closed, E. R. Hedrick* reverses the process we have used and defines "neighborhood " in terms of "limit." Assuming a limit relation fulfilling the conditions prescribed by Fréchet, and using a sequential definition of limiting element, he postulates that the fundamental domain is compact and that the derived class of every subclass is closed. He later assumes the "enclosable property," which affords a set of subclasses subject to conditions that validate their use in the sense of neighborhood. The type of neighborhood thus available may be used to set up a new definition of limiting element, in which case the fundamental domain is found to have the sequential property, so that the sequential theory he secures for the domain thus conditioned is equivalent to a neighborhood theory based, in the usual manner, on the type of neighborhood his assumptions afford. In fact, a domain with the enclosable property leads, in a very obvious manner, to a system $(\mathfrak{B} ; \mathfrak{U} ; T)$ fulfilling all five of the postulates we have used for such a system.

UntverastTy of Missouri, April, 1913.

- Loc. cit., p. 285. 